being taken from the other schools and placed in those at Hanwell, it may be that whilst the mortality tables there continue unchanged, those of other schools whence they are taken may present even a more favourable aspect than they have hitherto done. In fact, the two cases are so different that no parallel can be drawn between them. All that is admissible is the question, Can we from these discover the different degrees of sickness and mortality between country pauper children and those in large towns?

I have not Mr. 'Tufnell's report at hand, but the entire deathrate at the Central London District Schools is rather under two per cent., including-not "subtracting," as Dr. Wilkinson appears to suppose, and as The Times" version would perhaps imply-those sent in only to die; and is based, moreover, upon the average number in the house, not the total number of children admitted during the year. This latter method of calculation, upon which Dr. Wilkinson has proceeded to work out an average mortality of one-quarter per cent., is manifestly inadmissible; but if applied to the Hanwell Schools, would show less than one per cent. of deaths.

I can bear willing testimony to Mr. Aubin's skilful treatment of such cutaneous diseases as itch, ringworm, and porrigo; but $I$ believe the secret of his treatment consisted in great atten tion to cleanliness, and, by frequent inspection of the children, detecting eruptions in their earliest stage, and curing them before they could be communicated to other children-a degree of vigilance which it appears impossible to instil into the nurses.

Having already written a longer letter than I intended, I am unwilling to trespass further upon your space, but hope some day to forward many interesting particulars connected with the management of the poor children in these schools, which present perhaps one of the largest fields for the observation of struma in its various forms to be met with anywhere. I am, Sir, yours faithfully, JoHN Goodchito, L.R.C.P.

Ealing, June, 1861. Surgeon to the Central London District Schools, Hanwell.

\section{ACTION OF THE POTASSA FUSA.}

To the Editor of The LanceT.

Sir,-I shall feel much obliged by the insertion in your journal of a few brief remarks in reply to a passage relating to myself in Dr. Tilt's valuable practical observations on the "Use of Potassa Fusa cum Calce and of Potassa Canstica," which appeared in the last week's number of THE LANCET.

In the observations to which I refer, Dr. Tilt makes the following statement:- "Potassa fusa c. calce applied to the neck of the womb has a strange depressing influence on some patients, and without this exhaustion being cansed by pain. I have known this loss of power produce fainting, and to last more or less for two or three days. This stupefying influence of potassa fusa c. calce has been noted by Prof. Rust, of Berlin; and something similar has been observed by Mr. Wade, who insists on the sedative action of even the small quantities of potassa fusa, which he places in contact with the inodular tissues of confirmed urethral strictures, and on the caustic diminishing the patient's liability to retention of urine."

If Dr. Tilt will do me the favour to refer to my work on "Stricture and its Complications," he will find that the remarks of mine to which he alludes relate exclusively to the local action of the potassa fusa on urethral obstructions, and not to any constitutional effects of the remedy. Never having witnessed any constitutional effects from the use of potassa fusa in urethral stricture, similar to those described by Dr. Tilt as having occurred in some few instances from the application of the caustic potash to the neck of the uterus, I have thought it worth while to correct the error into which that gentleman has unintentionally fallen.

I am, Sir, your obedient servant,

Dean-street, Soho-square, June, 1861. R. WADE, F.R.C.S.

\section{PARISIAN MEDICAL INTELLIGENCE.}

(FROM OUR SPECIAL CORRESPONDENT.)

M. SALES-GIRoNs, the zealous promoter of a new method for the treatment of pulmonary complaints by the inhalation of minutely subdivided, or (as the technical expression is) " $p u l$ verized" liquids, does not seem destined to have it all his own way. Not only does his original opponent on this question, M. Champouillon, of the Val-de-Grâce, hold his ground very firmly, but now two other and equally redoubtable antagonists appear in the field. M.M. Pietra-Santa and Réné Brian, armed with weapons of a formidable nature in the shape of facts deduced from experiment, bid fair to overthrow the somewhat hasty conclusions of M. Sales.Girons, and to crush the nascent popularity of his pulverizing machine. A memoir of Dr. PietraSanta, just published, details the particulars of a course of in. vestigation, conducted with impartiality as it would appear, and wholly with the view of ascertaining the practical value of this soi-disant addition to therapeutics. The facts detailed in this paper seem to afford very decided answers to the three following important questions :-1 - st. Whether the pulverization of a mineral water does or does not affect the integrity of its chemical ingredients? Next, whether the particles of sub. divided water do or do not penetrate into the bronchi? And thirdly, whether the precise therapeutic effects of this new system of inhalation, supposing such to exist, admit of gradua. tion? M. Pietra-Santa commenced his experiments at the Eaux-bonnes, and ascertained that during the process of pulverization, the water of that thermal source speedily lost all its sulphuretted hydrogen, as well as the greater part of its sulphuret of sodium, sustaining, at the same time, a diminution of temperature varying from $25^{\circ}$ to $30^{\circ} \mathrm{Fahr}$. The mineral ingredients which predominate in the waters of the Eaux-bonnes are the sulphuret and chloride of sodium, and the addition of a solution of nitrate of silver occasions in them a copious precipitate of the white chloride and black sulphuret of silver. A similar reaction also takes place in the urine of patients drinking even small quantities of this water; but no such chemical changes have been obtained by similar treatment of the urine of persons who have inhaled only the pulverized fluid; the last fact proving either that none had penetrated into the bronchi, or else that absorption by the pulmonary mucous mem. brane had not occurred. Furthermore, from observation of the comparative results obtained by inhalation and by internal administration, (by drinking, it was apparent that the therapeutical effect had, in the former case, been negative or imper. ceptible, and in the latter, positively beneficial. M. Brian, who equally contests the validity of ir. Sales Girons' method, has published, in the Guzette Hebdomadaire, a series of observations, both clinical and experimental, in connexion with the subject, and his evidence is even more conclusive than that of his collaborateur. "The air-tubes," says this physiologist, "are organized exclusively for the passage of air, and whaterer else enters the lung, in howsoever small a quantity it be, that foreign element acts as an irritant, and produces cough. If, consequently, the smallest particle of medicated fluid does by chance reach the bronchi, a spasmodic and expulsive effort is most certain to ensue." In order to procure a practical solution of the doubt, M. Brian performed the following experiment in the laboratory of the College of France, in presence of MM. Claude Bernard, Vella, and Lecomte. He caused a small dog to inhale, during the space of half an hour, a solution of perchloride of iron in water pulverized secundum artem. At the expiration of the period, the animal was pithed, and its respiratory organs were carefully removed from the body, and some of the larger air-passages laid open. The mucous membranes of the larynx, trachea, and bronchi were then brushed over with a solution of cyanide of potassium, and the reagent was kept in contact with the tissue for the space of a quarter of an hour, without the production of any change of colour. The same test, applied to the mucons lining of the mouth, fauces, and nasal cavities, evinced, by the production of a well-marked blue colour, the presence of a certain quantity of perchloride of iron, sufficiently proving that the pulverized fluid had never reached the special air-passages. Two similar experiments, the one on a large dog, the other on a horse, were productive of like negative results. Not less conclusive than the foregoing is M. Champouillon's clinical test. This practitioner selected from his wards a man suffering from catarrhal bronchitis; he subjected this patient to a half hour's inhalation of a similarly charged solution, and after a preliminary rinsing of the mouth, tested with care the first expectorations, none of which gave the slightest indications of the presence of the perchloride; all of which form tolerably convincing proofs of the inutility of this much-vaunted method. I have been more prolix on this subject than may seem justifiable, but believing that to the discovery of a truth the detection of what is untrue ranks second only in a scientific point of view, I have been led to give your readers all the leading features of the adverse evidence.

At the Academy of Medicine last Tuesday, M. Littré read a paper, "On the Antiquity of Diphtheritic Epidemics," and made out a title of priority very decidedly in favour of Hippocrates. "In the sixth book of the 'Epidemics," we read," 